\title{
Normalization of Hamiltonian and nonlinear stability of triangular equilibrium points in the photogravitational restricted three body problem with P-R drag in non-resonance case
}

\author{
Ram Kishor \\ Central University of Rajasthan, NH-8, Bandarsindari, Kishangarh, Ajmer-305801, Rajasthan, India \\ kishor.ram888@gmail.com; kishor_math@curaj.ac.in \\ M. Xavier Rames Raj \\ Applied Mathematics Division, Vikram Sarabhai Space Centre, Thiruvananthapuram-695022, India \\ xavierjamesraj@gmail.com \\ Bhola Ishwar \\ Department of Mathematics, B. R. A. Bihar University, Muzaffarpur-842001, India \\ ishwar_bhola@hotmail.com
}

\begin{abstract}
Normal forms of Hamiltonian are very important to analyze the nonlinear stability of a dynamical system in the vicinity of invariant objects. This paper presents the normalization of Hamiltonian and the analysis of nonlinear stability of triangular equilibrium points in non-resonance case, in the photogravitational restricted three body problem under the influence of radiation pressures and P-R drags of the radiating primaries. The Hamiltonian of the system is normalized up to fourth order through Lie transform method and then to apply the Arnold-Moser theorem, Birkhoff normal form of the Hamiltonian is computed followed by nonlinear stability of the equilibrium points is examined. Similar to the case of classical problem, we have found that in the presence of assumed perturbations, there always exists one value of mass parameter within the stability range at which the discriminant $D_{4}$ vanish, consequently, Arnold-Moser theorem fails, which infer that triangular equilibrium points are unstable in nonlinear sense within the stability range. Present analysis is limited up to linear effect of the perturbations, which will be helpful to study the more generalized problem.
\end{abstract}

Subject headings: Normalization of Hamiltonian :Nonlinear stability :Non-resonance case :Restricted three body problem :Poynting-Roberston drag.

\section{Introduction}

Since the time of Poincaré, invariant objects are very much important to understand the behavior of a dynamical system, especially, phase space. Moreover, there are many possible approaches to find the invariant objects, whereas the normal forms (truncated) are very useful because these can give integrable approximations to the dynamics under appropriate hypothesis Jorba 1999). Because of the approximation of true dynamics by the normal forms, invariant objects of the initial system get approximated also, accordingly (Simó et al 1995; Jorba and Villanueva 1998). The approximate first integrals are those quantities, which are almost preserved through the system's flow. This shows that the surface levels by the flow are almost invariant. Some informa- 
tions about the dynamics can be obtained through this property. To minimize the overflow and complexity in the computations, an appropriate approach is to use of power series or Fourier sires, or a combination of both to represent the object. Because in many cases they needed only a few numbers of terms to maintain the good accuracy. Some other approach can also be found in Gómez et al (1998); Jorba and Masdemont (1999), in which trigonometric series is used. The normal forms of the Hamiltonian system up to some finite order is necessary to study the nonlinear stability of the equilibrium points using Arnold-Moser theorem in non-resonance case. They also help to know the behavior of dynamics in the neighborhood of the invariant objects. Many researchers have described the different method to find the normal forms of the Hamiltonian of the dynamical system (Poincaré 1881; Birkhoff 1927; Deprit 1969; Ushiki 1984; Coppola and Rand 1989a; Jorba 1999; Kishor and Kushvah 2017). In the normal forms, the central idea is to find suitable transforms of the phase co-ordinates, which can convert the Hamiltonian system in its simplest form up to a finite order of accuracy. Normalization of Hamiltonian is obtained to change the Hamiltonian into its simplest form using the method of Lie transforms (Coppola and Rand 1989a; Jorba 1999).

Because of radiating primary in the present problem under the analysis, force due to radiation pressure came into existence (Schuerman 1980; Ragos and Zagouras 1993), which acts in opposite direction to the gravitational attraction force of the primary. Concept of Poynting-Roberston drag is came into the picture when, Poynting (1903) investigated the effect of radiation pressure on the moving particle in interplanetary space and Robertson (1937) modified the Poynting's theory through the principle of relativity. In the analysis of Roberston, he considered only first order terms in the expression related to the ratio of velocity of the particle to that of the light. The radiation force is expressed as

$$
\vec{F}=F_{p}\left(\frac{\vec{R}}{R}-\frac{\vec{V} \cdot \vec{R} \vec{R}}{c R^{2}}-\frac{\vec{V}}{c}\right),
$$

where $F_{p}$ is the radiation pressure force due to radiating primary; $\vec{R}$ is the position vector of the particle relative to the radiating primary; $\vec{V}$ is the velocity of the particle; and $c$ is the speed of the light. First term of the equation (10) denotes the radiation pressure, second term represents the Doppler shift due to the motion of the particle, whereas third term corresponds to the absorption and subsequent re-emission part of induced radiation. The combined form of the last two terms of the equation (11) known as PoyntingRobertson (P-R) drag. Chernikov (1970) analyzed the photogravitational restricted three body problem (RTBP) with P-R drag under the frame of Sun-planet-particle system and found that noncollinear (triangular) equilibrium points are unstable. Effect of $\mathrm{P}-\mathrm{R}$ drag including radiation pressure is described by Schuerman (1980). A similar analysis is presented by Murray (1994) and Ragos and Zafiropoulos (1995) to observed the effect of P-R drag in the context of existence and stability of the equilibrium points. Kushvah et al (2007) examined the nonlinear stability in the generalized photogravitational RTBP with P-R drag of first primary and oblateness of secondary and found that triangular equilibrium points are unstable, whereas Mishra and Ishwar (2016) investigated about the stability of non-collinear equilibrium points in the photogravitational elliptic RTBP with P-R drag. Kushvah et al (2012) and Kishor and Kushvah (2013) have analyzed the effect of radiation pressure force on the existence and linear stability of the equilibrium points in the generalized photogravitational Chermnykh-like problem with a disc. They found that the effect of perturbation factors are significant. In literature, many researchers have analyzed the photogravitational RTBP in nonlinear sense by considering one or two perturbations at a time (McKenzie and Szebehely 1981; Ishwar 1997; Subba Rao and Krishan Sharma 1997; Lhotka and Celletti 2015; Alvarez-Ramírez et al 2015) but very few of them have considered the problem under the combined influence of few perturbations (Kushvah et al 2007; Kishor and Kushvah 2017). Ishwar and Sharma (2012) have discussed about the nonlinear stability of out of plane equilibrium points in the RTBP with oblate primary and found that $L_{6}$ point is stable in nonlinear sense. Raj and Ishwar (2017) have obtained diagonalized form of the Hamiltonian with P-R drag. Kishor and Kushvah (2017) have studied nonlinear stability of triangular equilibrium points in the Chermnykh-like problem, in the presence of 
radiation pressure, oblateness and a disc. They found that these perturbations affect the numerical results significantly.

Due to above reasons in addition to wide applications of the RTBP in mission design, we are motivated to study the problem under the influence of the radiation pressures and P-R drags of both primary and secondary. In the present study, we are interested to compute the fourth order normalized Hamiltonian and utilizing them to analyze the nonlinear stability of triangular equilibrium points using Arnold-Moser theorem in nonresonance case. Because of both primary and secondary radiating, the problem under analysis includes the four perturbing parameters in the form of mass reduction factors $q_{1}, q_{2}$ due to the radiation pressures of the primaries and P-R drags $W_{1}, W_{2}$ of both the primaries, respectively. The paper is organized as follows: In Section-2 we have formulated the problem and found the equations of motion. Section-3 presents the second order normalized Hamiltonian of the problem under analysis. Nonlinear stability analysis is discussed in Section-4. Section-5 is devoted to Birkhoff normal form and application of Arnold-Moser theorem in non-resonance case. Results are concluded in Section-6. For algebraic and numerical computations, Mathematica@(Wolfram 2003) software package is used. The results of this study may be used to describe more generalized problem under the influence of other perturbations such as albedo, solar wind drag, Stokes drag etc. (Idrisi and Ullah 2018; Singh and Omale 2019).

\section{Mathematical Formulation}

We consider the photogravitational restricted three body problem with P-R drag, which consists of motion of an infinitesimal mass under the influence of gravitational field and radiation effect of two massive and radiating bodies of masses $m_{1}$ and $m_{2},\left(m_{1}>m_{2}\right)$, respectively, called primaries. Forces, which govern the motion of infinitesimal mass are gravitational attractions, radiation pressures and P-R drags of both the primaries, respectively. It is assumed that gravitational effect of infinitesimal mass on the system is negligible. Units are normalized such as units of mass and distance are taken as the sum of the masses of both the primaries and separation dis- tance between them, respectively, whereas unit of time is the time period of the rotating frame. We suppose that the coordinate of the primaries are $(-\mu, 0),(1-\mu, 0)$, respectively and that of infinitesimal mass is $(x, y)$, then the equations of motion (Raj and Ishwar 2017) are

$$
\begin{aligned}
& \ddot{x}-2 \dot{y}=\frac{\partial U}{\partial x}, \\
& \ddot{y}-2 \dot{x}=\frac{\partial U}{\partial y},
\end{aligned}
$$

where

$$
\begin{aligned}
\frac{\partial U}{\partial x}= & x-\frac{q_{1}(1-\mu)(x+\mu)}{r_{1}^{3}}- \\
& \frac{q_{2}(1-\mu)(x+\mu-1)}{r_{2}^{3}}- \\
& \frac{W_{1} S_{1}}{r_{1}^{2}}-\frac{W_{2} S_{2}}{r_{2}^{2}} \\
\frac{\partial U}{\partial y}= & y-\frac{q_{1}(1-\mu) y}{r_{1}^{3}}-\frac{q_{2}(1-\mu) y}{r_{2}^{3}} \\
& -\frac{W_{1} S_{3}}{r_{1}^{2}}-\frac{W_{2} S_{4}}{r_{2}^{2}}
\end{aligned}
$$

and further

$$
\begin{aligned}
S_{1} & =\frac{(x+\mu)\{(x+\mu) \dot{x}+y \dot{y}\}}{r_{1}^{2}}+\dot{x}-y, \\
S_{2} & =\frac{(x+\mu-1)\{(x+\mu-1) \dot{x}+y \dot{y}\}}{r_{2}^{2}}+\dot{x}-y, \\
S_{3} & =\frac{y\{(x+\mu) \dot{x}+y \dot{y}\}}{r_{1}^{2}}+\dot{y}+x+\mu, \\
S_{4} & =\frac{y\{(x+\mu-1) \dot{x}+y \dot{y}\}}{r_{2}^{2}}+\dot{y}+x+\mu-1
\end{aligned}
$$

with $q_{i}=1-F_{p i} / F_{g i}, i=1,2$ as mass reduction factors of both the primaries, respectively; $F_{p i}, F_{g i}, i=1,2$-are the forces of radiation pressure and gravitational attraction of the respective primaries; $W_{1}=\left[\left(1-q_{1}\right)(1-\mu)\right] / c_{d}$ and $W_{2}=\left[\left(1-q_{2}\right) \mu\right] / c_{d}$ as P-R drags of both the primaries, respectively; $c_{d}$-is the speed of light in non-dimensional form; $r_{1}, r_{2}$ - are distances of infinitesimal mass from the first and second primary, which are given as

$r_{1}^{2}=(x+\mu)^{2}+y^{2}, \quad r_{2}^{2}=(x+\mu-1)^{2}+y^{2}$.

The co-ordinates $\left(x_{0}, \pm y_{0}\right)$ of triangular equilibrium points $L_{4,5}$ are obtained on similar basis as 
in Raj and Ishwar (2017). To overcome the complexity in the analysis, co-ordinates $x_{0}$ and $y_{0}$ are linearized with respected to $W_{1}, W_{2}, \epsilon_{1}, \epsilon_{2}$, keeping in mind that the perturbing parameters lie in $(0,1)$ so, take $q_{1}=1-\epsilon_{1}, q_{2}=1-\epsilon_{2}$, where $\epsilon_{1}, \epsilon_{2}$ are very small. The linearized co-ordinates $x_{0}$ and $y_{0}$ are

$$
\begin{aligned}
x_{0}= & \frac{1}{2}-\mu-\frac{4 W_{1}(2-\mu)}{3 \sqrt{3}}- \\
& \frac{4 W_{2}(1+2 \mu)}{3 \sqrt{3}}-\frac{\epsilon_{1}}{3}+\frac{\epsilon_{2}}{3} \\
y_{0}= \pm & \pm\left[\frac{\sqrt{3}}{2}+\frac{4 W_{1}(2-3 \mu)}{9}+\right. \\
& \left.\frac{4 W_{2}(1-3 \mu)}{9}-\frac{\epsilon_{1}}{3 \sqrt{3}}-\frac{\epsilon_{2}}{3 \sqrt{3}}\right] .
\end{aligned}
$$

The plus sign corresponds to $L_{4}$, whereas minus sign corresponds to $L_{5}$. The Hamiltonian function of the problem is written as

$$
\begin{aligned}
H= & p_{x} \dot{x}+p_{y} \dot{y}-\frac{\dot{x}^{2}+\dot{y}^{2}}{2}-\frac{x^{2}+y^{2}}{2}- \\
& x \dot{y}+\dot{x} y-\frac{(1-\mu) q_{1}}{r_{1}}-\frac{\mu q_{2}}{r_{2}}- \\
& W_{1} S_{5}-W_{2} S_{6},
\end{aligned}
$$

where

$$
\begin{aligned}
& S_{5}=\frac{(x+\mu) \dot{x}+y \dot{y}}{2 r_{1}^{2}}-\arctan \left(\frac{y}{x+\mu}\right), \\
& S_{6}=\frac{(x+\mu-1) \dot{x}+y \dot{y}}{2 r_{1}^{2}}-\arctan \left(\frac{y}{x+\mu-1}\right) .
\end{aligned}
$$

The conjugate momenta $p_{x}, p_{y}$ corresponding to generalized co-ordinate $x, y$ respectively, are given as

$$
\begin{aligned}
& p_{x}=\dot{x}-y+\frac{W_{1}(x+\mu)}{2 r_{1}^{2}}+\frac{W_{2}(x+\mu-1)}{2 r_{2}^{2}}(1,0) \\
& p_{y}=\dot{y}+x+\frac{W_{1} y}{2 r_{1}^{2}}+\frac{W_{2} y}{2 r_{2}^{2}} .
\end{aligned}
$$

\section{Second Order Normal Form of the Hamiltonian}

In the present analysis only the stability of $L_{4}$ is analyzed, because the dynamics of $L_{5}$ is similar to that of $L_{4}$. Only first order terms in the perturbing parameters $W_{1}, W_{2}, q_{1}, q_{2}$ are considered for simplifying the complex calculations involved in the problem through out the analysis. The second order normal form of the Hamiltonian of the problem under analysis is obtained in Raj and Ishwar (2017) and for self sufficiency of this paper, we have taken some necessary expressions in appropriate form there to use under this section. Shifting the origin to the triangular equilibrium point $L_{4}$ using simple transformations as

$$
\begin{aligned}
& x^{*}=x-x_{0}, y^{*}=y-y_{0}, \\
& p_{x}{ }^{*}=p_{x}+y_{0}, p_{y}{ }^{*}=p_{y}-x_{0} .
\end{aligned}
$$

Substituting these variables in Hamiltonian (9), we get new Hamiltonian $H^{*}$. Now, expanding the new Hamiltonian using Taylor's series about the origin, which is now, the triangular equilibrium point, $H^{*}$ can be written as

$H^{*}=H_{0}^{*}+H_{1}^{*}+H_{2}^{*}+H_{3}^{*}+\cdots+H_{n}^{*}+\ldots$,

where

$$
H_{n}^{*}=\sum H_{i j k l} x^{* i} y^{* j} p_{x}{ }^{* k} p_{y}{ }^{* l},
$$

such that $i+j+k+l=n$. Since, the origin is the triangular equilibrium point, $H_{1}^{*}$ must vanish, whereas $H_{0}^{*}$ is constant hence, it can be dropped out as it is irrelevant to the dynamics. The quadratic Hamiltonian $H_{2}^{*}$, which is to be normalized first and then to be used for higher order normalization, is given as

$$
\begin{aligned}
H_{2}^{*}= & \frac{p_{x} *^{2}+p_{y}{ }^{2}}{2}+y^{*} p_{x}{ }^{*}-x^{*} p_{y}{ }^{*} \\
& +E x^{* 2}+G x^{*} y^{*}+F y^{* 2},
\end{aligned}
$$

where

$$
\begin{aligned}
E= & \frac{1}{8}+\frac{4 W_{1}}{\sqrt{3}}+\frac{2 W_{1}}{\sqrt{3}}+\frac{\epsilon_{1}}{4}-\frac{\epsilon_{2}}{2},(15) \\
F= & -\frac{5}{8}-\frac{4 W_{1}}{\sqrt{3}}-\frac{2 W_{1}}{\sqrt{3}}-\frac{\epsilon_{1}}{4}+\frac{\epsilon_{2}}{2}(16) \\
G= & -\gamma\left(1-\frac{32 W_{1}}{9 \sqrt{3}}-\frac{16 W_{1}}{9 \sqrt{3}}\right. \\
& \left.-\frac{2 \epsilon_{1}}{9}+\frac{4 \epsilon_{2}}{9}\right), \\
\text { with } \gamma= & \frac{3 \sqrt{3}}{4}(1-2 \mu) .
\end{aligned}
$$

In the present study, the problem is dealt with four perturbation parameters in the form of $\mathrm{P}$ $\mathrm{R}$ drag and radiation pressure of both the primaries. Hence, the coefficient $H_{i j k l}$ for $i, j, k, l=$ 
$0,1,2,3,4$ such that $i+j+k+l=4$ in (13) can be bifurcated into five parts such as $H_{i j k l 1}, H_{i j k l 2}, H_{i j k l 3}, H_{i j k l 4}$, and $H_{i j k l 5}$, which corresponds to the terms in classical case, terms with P-R drag of first primary $W_{1}$, P-R drag of second primary $W_{2}$, radiation pressure of first primary $\epsilon_{1}=1-q_{1}$ and radiation pressure of second primary $\epsilon_{2}=1-q_{2}$, respectively. Thus,

$$
\begin{aligned}
H_{i j k l}= & H_{i j k l 1}+H_{i j k l 2}+H_{i j k l 3} \\
& +H_{i j k l 4}+H_{i j k l 5} .
\end{aligned}
$$

It is noted that if there is no perturbations in the system, i.e. $W_{1}=W_{2}=\epsilon_{1}=\epsilon_{2}=0$, then $H_{i j k l}=$ $H_{i j k l 1}$, which is nothing but the coefficient of the Hamiltonian in classical case.

Hamiltonian equations of motion of the infinitesimal mass in matrix form is written as

$$
\left[\begin{array}{c}
\dot{x^{*}} \\
\dot{y}^{*} \\
\dot{p_{x}} \\
\dot{p}_{y}^{*}
\end{array}\right]=\left[\begin{array}{cccc}
0 & 1 & 1 & 0 \\
-1 & 0 & 0 & 1 \\
-2 E & -G & 0 & 1 \\
-G & -2 F & -1 & 0
\end{array}\right]\left[\begin{array}{c}
x^{*} \\
y^{*} \\
p_{x}{ }^{*} \\
p_{y}{ }^{*}
\end{array}\right] \text {. }
$$

The characteristic equation of the system (20) is

$$
\begin{aligned}
& \lambda^{4}+2(E+F+1) \lambda^{2}+\left(4 E F-G^{2}-\right. \\
& 2 E-2 F+1)=0 .
\end{aligned}
$$

Solving the simplified discriminant of the characteristic equation (21) as

$$
(E+F+1)^{2}-\left(4 E F-G^{2}-2 E-2 F+1\right)=0,
$$

we have the value of critical mass ratio $0<\mu_{c} \leq$ $(1 / 2)$ as

$$
\begin{aligned}
\mu_{c}= & 0.0385209+0.0823761 W_{1}+0.0823761 W_{2} \\
& +0.0178349 \epsilon_{1}-0.356699 \epsilon_{2},
\end{aligned}
$$

which is similar to that of Kushvah et al (2007) and Kishor and Kushvah (2013) and agree with the classical value $\mu_{c}=0.0385209$. Figure (1) (a-d) shows the variations of critical mass ratio $\mu_{c}$ with respect to perturbing parameters $W_{1}, W_{2}, \epsilon_{1}$ and $\epsilon_{2}$, respectively. We observed that the effects of the perturbations in question are significant. As, system will be stable when four roots of the characteristic equation (21) are pure imaginary, which is possible when the mass parameter $\mu$ satisfy the condition $0<\mu<\mu_{c}$. Since, we are analyzing the nonlinear stability within the range of linear
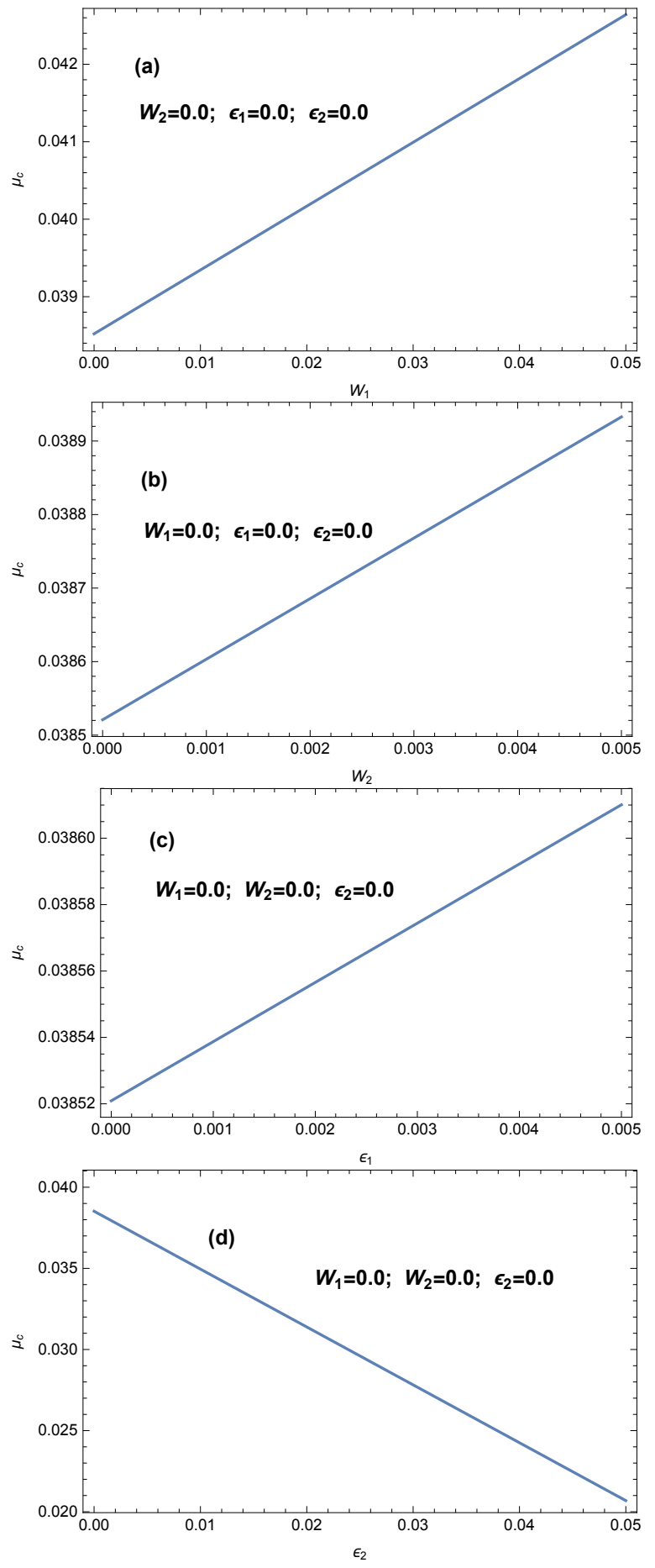

Fig. 1. - Variation of critical mass ratio $\mu_{c}$ with respect to (a) $W_{1}$, (b) $W_{2}$, (c) $\epsilon_{1}$ and (d) $\epsilon_{2}$. 
stability $0<\mu<\mu_{c}$, it is obvious to assume that roots of the characteristic equation (21) are pure imaginary. Suppose, the roots of the characteristic equation (21) are $\pm i \omega_{1}$ and $\pm i \omega_{2}$, where $\omega_{1}, \omega_{2}$ can be obtained by solving the equation

$$
\begin{aligned}
& \omega^{4}-2(E+F+1) \omega^{2}+\left(4 E F-G^{2}-\right. \\
& 2 E-2 F+1)=0 .
\end{aligned}
$$

Motion corresponds to frequencies $\omega_{1}, \omega_{2} \in \mathbb{R}$ are known as long and short periodic motion of infinitesimal mass at $L_{4}$ with periods of $2 \pi / \omega_{1}$ and $2 \pi / \omega_{2}$, respectively. Frequencies $\omega_{1}, \omega_{2}$ corresponding to the long and short periodic motion are related to each other by the means of relations

$$
\begin{aligned}
\omega_{1}^{2}+\omega_{2}^{2} & =2 E+2 F+2 \\
\omega_{1}^{2} \omega_{2}^{2} & =4 E F-G^{2}-2 E-2 F+1 .
\end{aligned}
$$

Substituting the values of $E, F$ and $G$ from equations (15)[17), we get

$$
\begin{aligned}
\omega_{1}^{2}+\omega_{2}^{2}= & 1, \\
\omega_{1}^{2} \omega_{2}^{2}= & \frac{27}{16} \gamma^{2}-4 \sqrt{3} W_{1}-2 \sqrt{3} W_{2}- \\
& \frac{2 \epsilon_{1}}{3}+\frac{3 \epsilon_{2}}{4},
\end{aligned}
$$

where the values of $\omega_{1}$ and $\omega_{2}$ are

$\omega_{1}=\sqrt{-1+\sqrt{1-4 \delta}}, \quad \omega_{2}=\sqrt{-1-\sqrt{1-4 \delta}}$,

with

$\delta=\frac{27}{16}-\gamma^{2}-4 \sqrt{3} W_{1}-2 \sqrt{3} W_{2}-\frac{3 \epsilon_{1}}{4}+\frac{3 \epsilon_{2}}{2}$.

The real normalized Hamiltonian of the Hamiltonian (14) up to second order is given as (Raj and Ishwar 2017)

$$
H_{2}=\omega_{1} \frac{\mathbf{x}^{2}+\mathbf{p}_{\mathbf{x}}^{\mathbf{2}}}{2}+\omega_{2} \frac{\mathbf{y}^{2}+\mathbf{p}_{\mathbf{y}}^{\mathbf{2}}}{2},
$$

which is complexified by using the co-ordinate transformations

$$
\begin{aligned}
\mathbf{x} & =\frac{X+i P_{X}}{\sqrt{2}}, \\
\mathbf{y} & =\frac{-Y+i P_{Y}}{\sqrt{2}}, \\
\mathbf{p}_{\mathbf{x}} & =\frac{i X+P_{X}}{\sqrt{2}}, \\
\mathbf{p}_{\mathbf{y}} & =\frac{i Y-P_{Y}}{\sqrt{2}}
\end{aligned}
$$

and changed as

$$
H_{2}=i \omega_{1} X P_{X}-i \omega_{2} Y P_{Y},
$$

Finally, symplectic matrix $\mathbf{C}$ of the symplectic transformations, which are used to obtain the complex normal form of Hamiltonian is given as (Raj and Ishwar 2017)

$$
\mathbf{C}=\left[s_{i j}\right], 1 \leq i, j \leq 4
$$

with

$$
\begin{aligned}
& s_{11}=0=s_{12}, s_{13}=\frac{1-2 F+\omega_{1}^{2}}{\sqrt{d\left(\omega_{1}\right)}}, \\
& s_{14}=\frac{1-2 F+\omega_{1}^{2}}{\sqrt{d\left(\omega_{2}\right)}}, s_{21}=\frac{2 \omega_{1}}{\sqrt{d\left(\omega_{1}\right)}}, \\
& s_{22}=\frac{2 \omega_{1}}{\sqrt{d\left(\omega_{2}\right)}}, s_{23}=\frac{G}{\sqrt{d\left(\omega_{1}\right)}}, \\
& s_{24}=\frac{G}{\sqrt{d\left(\omega_{2}\right)}}, s_{31}=\frac{\omega_{1}^{3}-(2 F+1) \omega_{1}}{\sqrt{d\left(\omega_{1}\right)}}, \\
& s_{32}=\frac{\omega_{2}^{3}-(2 F+1) \omega_{2}}{\sqrt{d\left(\omega_{2}\right)}}, s_{33}=\frac{-G}{\sqrt{d\left(\omega_{1}\right)}}, \\
& s_{34}=\frac{-G}{\sqrt{d\left(\omega_{2}\right)}}, s_{41}=\frac{G \omega_{1}}{\sqrt{d\left(\omega_{1}\right)}}, s_{42}=\frac{G \omega_{2}}{\sqrt{d\left(\omega_{2}\right)}}, \\
& s_{43}=\frac{1-2 F-\omega_{1}^{2}}{\sqrt{d\left(\omega_{1}\right)}}, s_{44}=\frac{1-2 F-\omega_{2}^{2}}{\sqrt{d\left(\omega_{2}\right)}},
\end{aligned}
$$

where $d\left(\omega_{i}\right)$ for $i=1,2$ is obtained from the following equation

$$
\begin{aligned}
d(\omega)= & \omega\left[\omega^{4}-(2 E+6 F) \omega^{2}+\right. \\
& \left.\left(4 E F+4 F^{2}-2 E+2 F-2\right)\right] .
\end{aligned}
$$

\section{Nonlinear Stability in Non-resonance Case}

Nonlinear stability of the equilibrium points can be described in two cases, one as resonance case and other as non-resonance case. For resonance case, the nonlinear stability is studied through the theorems of Markeev and Sokolskii (1977) as in Goździewski (1998) and for nonresonance case, it is analyzed through the ArnoldMoser theorem. In the present analysis the nonlinear stability of the perturbed triangular equilibrium point in non-resonance case will be studied through Arnold-Moser theorem (Meyer and Schmidt 1986; Mever et al 1992), which is described as follows: 
Consider the Hamiltonian expressed in action variables $I_{1}, I_{2}$ and angles variables $\phi_{1}, \phi_{2}$ as,

$$
K=K_{2}+K_{4}+\cdots+K_{2 m}+K_{2 m+1},
$$

in which: (i) $K_{2 m}$ is homogeneous polynomial of degree $m$ in action variables $I_{1}, I_{2}$ and $K_{2 m+1}$ is higher degree polynomial than $m$ (ii) $K_{2}=\omega_{1} I_{1}-$ $\omega_{2} I_{2}$ with $\omega_{1,2}$ as positive constants (iii) $K_{4}=$ $-\left(A I_{1}^{2}+B I_{1} I_{2}+C I_{2}^{2}\right)$, where $A, B, C$ are constants to be determined. Since, $K_{2}, K_{4}, \ldots, K_{2 m}$ are functions of $I_{1}$ and $I_{2}$, the Hamiltonian (39) follows the Birkhoff normal form (Birkhoff 1927) up to the terms $m$. This can be obtained with some non-resonance condition on the frequencies $\omega_{1}, \omega_{2}$. To state the Arnold-Moser theorem, we assume that $K$ is in the required form.

Arnold-Moser Theorem: The origin is stable for the system whose Hamiltonian is (39) provided for some $\nu, 2 \leq \nu \leq m, D_{2 \nu}=$ $K_{2 \nu}\left(\omega_{2}, \omega_{1}\right) \neq 0$.

Since, for Arnold-Moser theorem, Birkhoff normal form of the Hamiltonian is necessary and for Birkhoff normal form, assumption of non-resonance on frequencies is required. The non-resonance condition of frequencies as in Deprit and Deprit-Bartholome (1967); Kishor and Kushvah (2017) is that if $\omega_{1}, \omega_{2}$ are frequencies of infinitesimal mass in linear dynamics and $\sigma \in \mathbb{Z}$ such that $\sigma \geq 2$, then

$$
\sigma_{1} \omega_{1}+\sigma_{2} \omega_{2} \neq 0
$$

for all $\sigma_{1}, \sigma_{2} \in \mathbb{Z}$ satisfying $\left|\sigma_{1}\right|+\left|\sigma_{2}\right| \leq 2 \sigma$. This is also, called as condition of irrationality, which insures that there exists a symplectic normalizing transformation which transform the Hamiltonian (12) in the form of Hamiltonian (39). Coefficients of the normalized Hamiltonian are independent on the integer $\sigma$ as well as to the transformation obtained. In specific

$$
\operatorname{det}\left|\begin{array}{ccc}
\frac{\partial^{2} K}{\partial I_{1}^{2}} & \frac{\partial^{2} K}{\partial I_{1} \partial I_{2}} & \frac{\partial K}{\partial I_{1}} \\
\frac{\partial^{2} K}{\partial I_{2} \partial I_{1}} & \frac{\partial^{2} K}{\partial I_{2}^{2}} & \frac{\partial K}{\partial I_{2}} \\
\frac{\partial K}{\partial I_{1}} & \frac{\partial K}{\partial I_{2}} & 0
\end{array}\right|_{I_{1}, I_{2}=0}
$$

is invariant of the Hamiltonian (39) with respect to the symplectic transformation considered. The nonlinear stability of perturbed triangular equilibrium points is analyzed through the Arnold-Moser theorem under these conditions. In classical case frequencies $\omega_{1}, \omega_{2}$ satisfy the condition $0<\omega_{2}<$ $(1 / \sqrt{2})<\omega_{1}<1$. Therefore, if $\sigma=2$, then irrationality condition (40) fails for following pairs of integers $\sigma_{1}=1, \sigma_{2}=-2, \sigma_{1}=-1, \sigma_{2}=2$, $\sigma_{1}=1, \sigma_{2}=-3$ and $\sigma_{1}=-1, \sigma_{2}=3$. First, two pairs of integers with condition (40) yield $\left(\omega_{1} / \omega_{2}\right)=(1 / 2)$ and last two pairs of integers give $\left(\omega_{1} / \omega_{2}\right)=(1 / 3)$, which are also known as second and third order resonance of the frequencies respectively. If $\left(\omega_{1} / \omega_{2}\right)=(1 / 2)$ or $\omega_{1}=2 \omega_{2}$, then from equations (27,28), we get

$$
\frac{4}{25}=\frac{27}{16} \gamma^{2}-4 \sqrt{3} W_{1}-2 \sqrt{3} W_{2}-\frac{2 \epsilon_{1}}{3}+\frac{3 \epsilon_{2}}{4} .
$$

Simplifying equation (42), we have a quadratic equation in $\mu$ as

$$
\begin{aligned}
& \frac{27}{16} \mu^{2}-\frac{27}{16} \mu+\left(\sqrt{3} W_{1}+\frac{\sqrt{3} W_{2}}{2}+\right. \\
& \left.\frac{3 \epsilon_{1}}{16}-\frac{3 \epsilon_{2}}{8}+\frac{1}{25}\right)=0 .
\end{aligned}
$$

The solution $\mu=\mu_{c 1}$ of equation (43) within the stability range $0<\mu<\mu_{c}$ is

$$
\begin{aligned}
\mu_{c 1}= & 0.0242939+1.078820 W_{1}+0.539409 W_{2} \\
& +0.116785 \epsilon_{1}-0.233571 \epsilon_{2} .
\end{aligned}
$$

This means, Arnold-Moser theorem fails at $\mu_{c 1} \in$ $\left(0, \mu_{c}\right)$. If $\left(\omega_{1} / \omega_{2}\right)=(1 / 3)$ or $\omega_{1}=3 \omega_{2}$, then proceeding on similar basis, we find that ArnoldMoser theorem fails at $\mu=\mu_{c 2}$, where

$$
\begin{aligned}
\mu_{c 2}= & 0.013516+1.054920 W_{1}+0.527459 W_{2} \\
& +0.114198 \epsilon_{1}-0.228396 \epsilon_{2} .
\end{aligned}
$$

Equations (44,45) are similar to that of the results in Deprit and Deprit-Bartholome (1967); Kishor and Kushvah (2013) and agree with classical result in the absence of perturbing parameters. To see the effects of perturbing parameters on $\mu_{c 1}$ and $\mu_{c 2}$, its numerical values are computed and presented in Table-1. From Table-1, it is clear that the values of $\mu_{c 1}$ and $\mu_{c 2}$ are very much affected from radiation pressures and P-R drags of the primaries.

\section{Fourth order Normalized Hamiltonian}

Since, Birkhoff's normal form up to fourth order of the Hamiltonian is necessary to apply the Arnold-Moser theorem, which is computed from second order normalized Hamiltonian 
(13) using Lie transform method described in Coppola and Rand (1988, 1989b); Jorba (1999); Celletti (2010); Kishor and Kushvah (2017). As, in the paper of Coppola and Rand (1989b) as well as in the book of Celletti (2010), higher order normalized Hamiltonian is

$$
K=K_{2}+K_{3}+K_{4}+\cdots+K_{n}+\ldots,
$$

where

$$
K_{n}=\sum K_{i j k l} X^{i} Y^{j} P_{X}^{k} P_{Y}^{l}
$$

such that $i+j+k+l=n$. Quadratic part of $K$ is $K_{2}=H_{2}$, whereas $K_{n}$ through the nth step of Lie transform is given as

$$
K_{n}=\frac{1}{n}\left\{H_{2}, G_{n}\right\}+(\text { known terms }),
$$

where Lie bracket of normalized quadratic Hamiltonian $\mathrm{H}_{2}$ and generating function $G_{n}$ is defined as

$$
\begin{aligned}
\left\{H_{2}, G_{n}\right\}= & \frac{\partial H_{2}}{\partial X} \frac{\partial G_{n}}{\partial P_{X}}-\frac{\partial H_{2}}{\partial P_{X}} \frac{\partial G_{n}}{\partial X} \\
& +\frac{\partial H_{2}}{\partial Y} \frac{\partial G_{n}}{\partial P_{Y}}-\frac{\partial H_{2}}{\partial P_{Y}} \frac{\partial G_{n}}{\partial Y} .
\end{aligned}
$$

Using $H_{2}$ from equation (13), it reduces to

$$
\begin{aligned}
\left\{H_{2}, G_{n}\right\}= & \mathbf{i} \omega_{1}\left(P_{X} \frac{\partial G_{n}}{\partial P_{X}}-X \frac{\partial G_{n}}{\partial X}\right) \\
& +\mathbf{i} \omega_{2}\left(P_{Y} \frac{\partial G_{n}}{\partial P_{Y}}-Y \frac{\partial G_{n}}{\partial Y}\right)
\end{aligned}
$$

The choice of generating function $G_{n}$ is such that the above partial differential operator on $G_{n}$ remove large possible number of terms from the expression of $K_{n}$. As, each terms of the $K_{n}$ is of the form $\alpha X^{i} Y^{j} P_{X}{ }^{k} P_{Y}{ }^{l}$, where $\alpha$ is constant, we can assume terms in $G_{n}$ of the form $\beta X^{i} Y^{j} P_{X}{ }^{k} P_{Y}{ }^{l}$, where constant $\beta$ is to be determined. Therefore, we obtain that

$$
\frac{\left\{H_{2}, G_{n}\right\}}{n}=\frac{\mathbf{i} \beta}{n}\left[(k-i) \omega_{1}-(l-j)\right] X^{i} Y^{j} P_{X}{ }^{k} P_{Y}{ }^{l},
$$

and hence,

$$
\beta=\frac{\mathbf{i} \alpha}{\left[(k-i) \omega_{1}-(l-j)\right]}, i+j+k+l=n .
$$

This shows that even in the non-resonance case, the term of the form $X^{i} Y^{j} P_{X}{ }^{i} P_{Y}^{j}$ in $K_{n}$ can not be deleted because of vanishing denominator in (52) at $i=k, j=l$, whereas in the resonance case some additional non-removable terms occur while solving the generating function $G_{n}$. Hence, in nonresonance case, the Hamiltonian of the present problem can be written in the form of (46), in which

$$
\begin{aligned}
K_{2} & =i \omega_{1} X P_{X}-i \omega_{2} Y P_{Y} \\
K_{3} & =0 \\
K_{4} & =\frac{A X^{2} P_{X}^{2}+B X P_{X} Y P_{Y}+C Y^{2} P_{Y}^{2}}{2}
\end{aligned}
$$

where $A=2 K_{2020}, B=2 K_{1111}$, and $C=2 K_{0202}$. Using action variables $I_{1}=i X P_{X}$ and $I_{2}=i Y P_{Y}$ in equations (5355), we get

$$
\begin{aligned}
& K_{2}=\omega_{1} I_{1}-\omega_{2} I_{2}, \\
& K_{3}=0, \\
& K_{4}=-\left(A I_{1}^{2}+B I_{I} 1_{2}+C I_{2}^{2}\right) .
\end{aligned}
$$

Thus, normalized Hamiltonian up to fourth order is

$$
\begin{aligned}
K\left(I_{1}, I_{2}\right)= & K_{2}+K_{3}+K_{4} \\
= & \omega_{1} I_{1}-\omega_{2} I_{2}-K_{2020} I_{1}^{2}+ \\
& K_{1111} I_{I} 1_{2}+K_{0202} I_{2}^{2},
\end{aligned}
$$

which agree with that of Deprit and Deprit-Bartholome (1967); Kushvah et al (2007); Kishor and Kushvah (2017).

Form equations (59), it is clear that fourth order normalized Hamiltonian is the function of only action variables $I_{1}, I_{2}$, which shows that these are in Birkhoff normal form. The coefficients $K_{i j k l}$ used in the equation (55) or 58) can be written into 5 parts such as $K_{i j k l 1}, K_{i j k l 2}, K_{i j k l 3}, K_{i j k l 4}$ and $K_{i j k l 5}$ for $i, j, k, l=0,1,2,3,4$ such that $i+j+k+l=4$. These coefficients corresponds to the term of classical part, terms with P-R drags $W_{1}$ and $W_{2}$ of first and second primary, radiation pressures $\epsilon_{1}=1-q_{1}$ and $\epsilon_{2}=1-q_{2}$ of first and second primary, respectively. In the absence of perturbing parameters i.e. for $W_{1}=W_{1}=\epsilon_{1}=$ $\epsilon_{2}=0, K_{i j k l}=K i j k l 1$. Therefore, $K_{2020}, K_{1111}$ 
Table 1: $\mu_{c 1}$ and $\mu_{c 2}$ at different values of perturbing parameters.

\begin{tabular}{|rrrrrr|}
\hline$W_{1}$ & $W_{1}$ & $\epsilon_{1}$ & $\epsilon_{2}$ & $\mu_{c 1}$ & $\mu_{c 2}$ \\
\hline 0.000 & 0.0000 & 0.000 & 0.00 & 0.024294 & 0.013516 \\
& & & & & \\
0.005 & 0.0000 & 0.000 & 0.00 & 0.029688 & 0.018791 \\
0.010 & 0.0000 & 0.000 & 0.00 & 0.035082 & 0.024065 \\
0.015 & 0.0000 & 0.000 & 0.00 & 0.040476 & 0.029340 \\
& & & & & \\
0.000 & 0.0040 & 0.000 & 0.00 & 0.026452 & 0.015626 \\
0.000 & 0.0045 & 0.000 & 0.00 & 0.026721 & 0.015890 \\
0.000 & 0.0050 & 0.000 & 0.00 & 0.026991 & 0.016153 \\
& & & & & \\
0.000 & 0.0000 & 0.001 & 0.00 & 0.024411 & 0.013630 \\
0.000 & 0.0000 & 0.002 & 0.00 & 0.024528 & 0.013744 \\
0.000 & 0.0000 & 0.003 & 0.00 & 0.024644 & 0.013859 \\
& & & & & \\
0.000 & 0.0000 & 0.000 & 0.01 & 0.021958 & 0.011232 \\
0.000 & 0.0000 & 0.000 & 0.02 & 0.019623 & 0.008948 \\
0.000 & 0.0000 & 0.000 & 0.03 & 0.017287 & 0.006664 \\
& & & & & \\
0.005 & 0.0040 & 0.001 & 0.01 & 0.029627 & 0.018731 \\
\hline
\end{tabular}
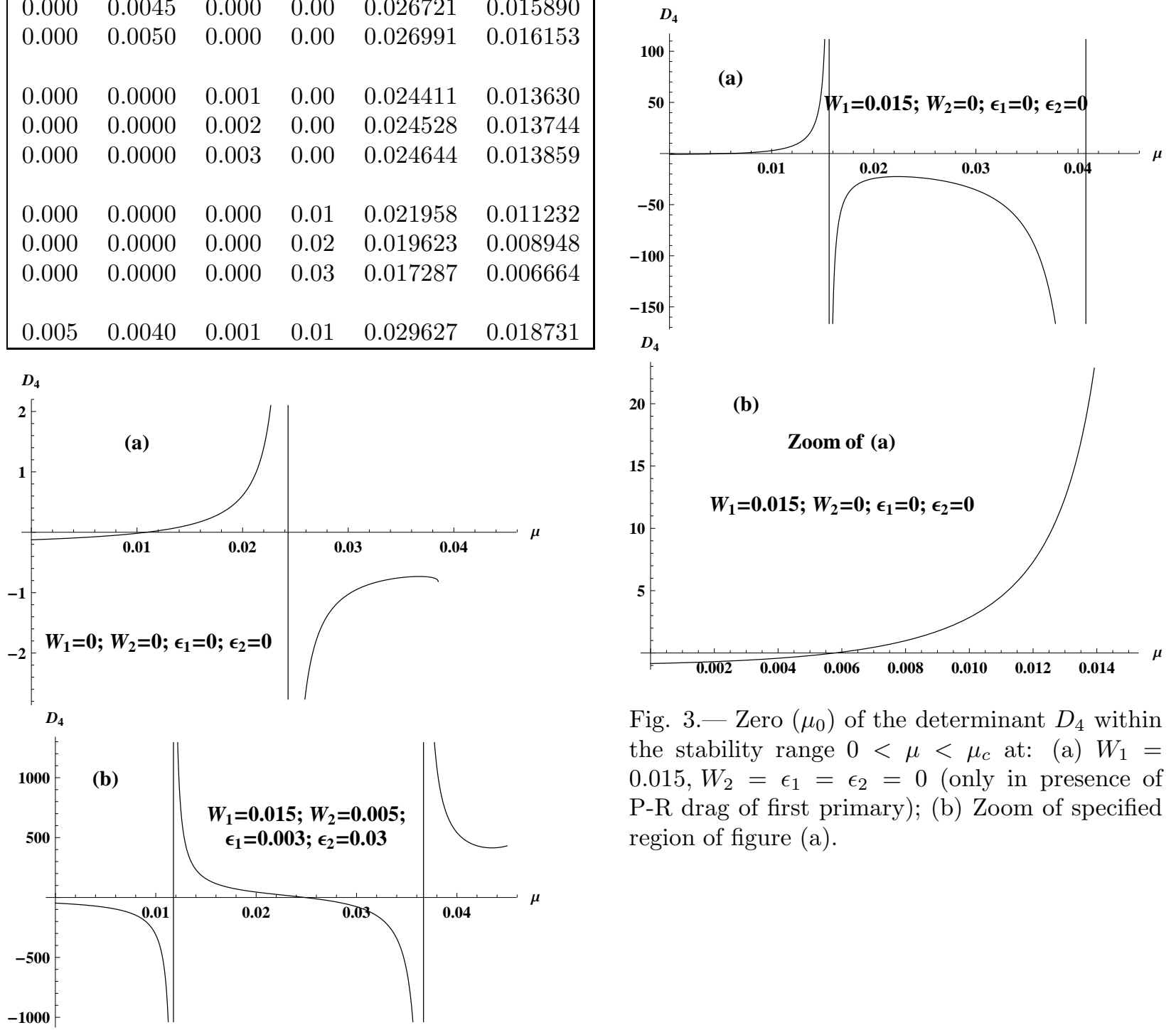

Fig. 3.- Zero $\left(\mu_{0}\right)$ of the determinant $D_{4}$ within the stability range $0<\mu<\mu_{c}$ at: (a) $W_{1}=$ $0.015, W_{2}=\epsilon_{1}=\epsilon_{2}=0$ (only in presence of P-R drag of first primary); (b) Zoom of specified region of figure (a).

Fig. 2.- Zero $\left(\mu_{0}\right)$ of the determinant $D_{4}$ within the stability range $0<\mu<\mu_{c}$ at: (a) $W_{1}=W_{2}=$ $\epsilon_{1}=\epsilon_{2}=0$ (classical case); (b) $W_{1}=0.015, W_{2}=$ $0.005, \epsilon_{1}=0.003, \epsilon_{2}=0.03$ (perturbed case). 


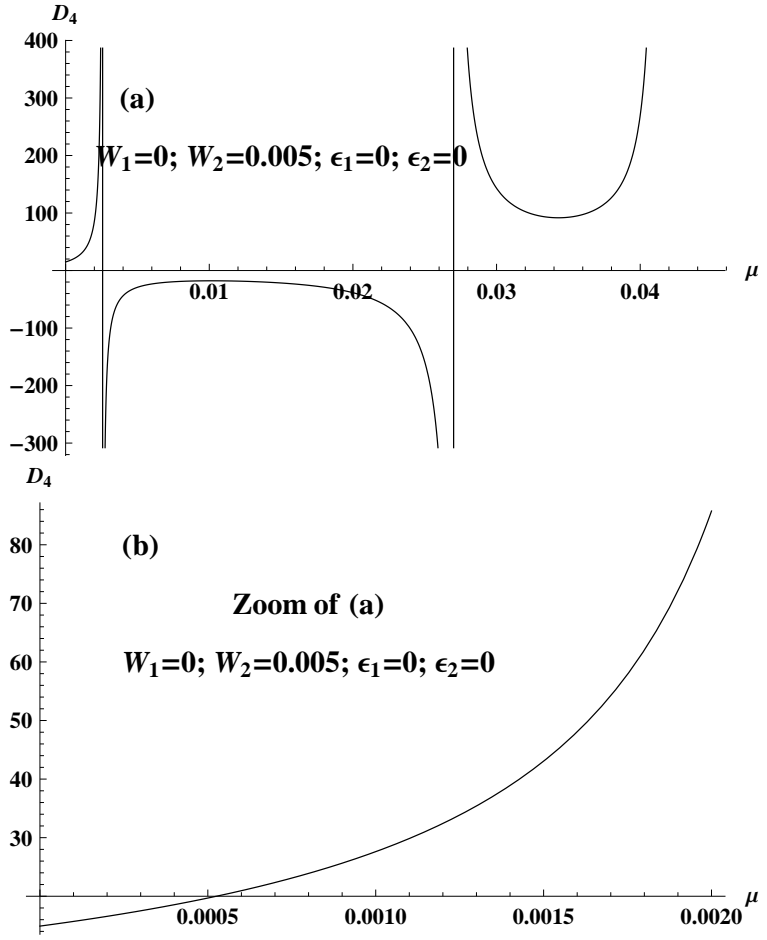

Fig. 4.- Zero $\left(\mu_{0}\right)$ of the determinant $D_{4}$ within the stability range $0<\mu<\mu_{c}$ at: (a) $W_{2}=$ $0.005, W_{1}=\epsilon_{1}=\epsilon_{2}=0$ (only in presence of P$\mathrm{R}$ drag of second primary); (b) Zoom of specified region of figure (a).

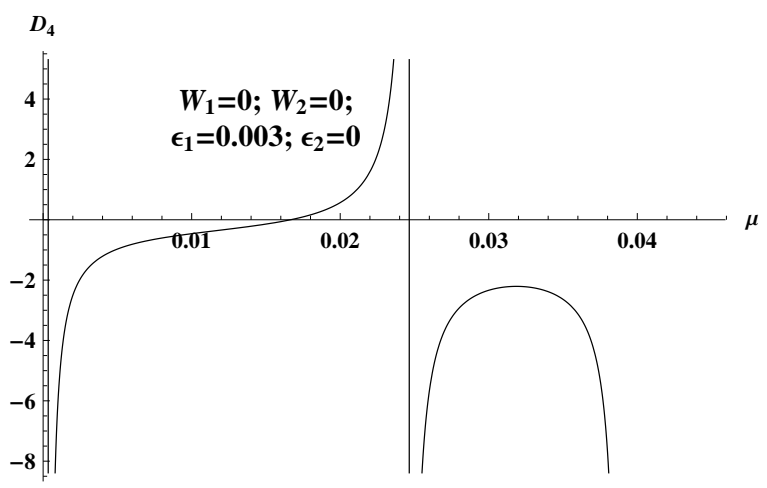

Fig. 5.- Zero $\left(\mu_{0}\right)$ of the determinant $D_{4}$ within the stability range $0<\mu<\mu_{c}$ at: $\epsilon_{1}=1-q_{1}=$ 0.003, $W_{1}=W_{2}=\epsilon_{2}=0$ (only in presence of radiation pressure of first primary). and $K_{0202}$ become

$$
\begin{aligned}
K_{2020}= & K_{20201}+K_{20202}+K_{20203}+ \\
& K_{20204}+K_{20205}, \\
K_{1111}= & K_{11111}+K_{11112}+K_{11113}+ \\
& K_{11114}+K_{11115} \\
K_{0202}= & K_{02021}+K_{02022}+K_{02023}+ \\
& K_{02024}+K_{02025} .
\end{aligned}
$$

The algebraic expressions of above 15 coefficients on right hand sides of equations 60, 62 are too complicated and huge to be placed here hence, we avoid to present in the paper. These are utilized to compute the determinant $D_{4}=K_{4}\left(\omega_{2}, \omega_{1}\right)$ for applying the Arnold-Moser theorem. For the simplicity, $D_{4}$ is expressed as

$$
\begin{aligned}
D_{4}= & \left(\frac{A_{1}}{B_{1}}\right)+\left(\frac{A_{2}}{B_{2}}\right) W_{1}+\left(\frac{A_{3}}{B_{3}}\right) W_{2}+ \\
& \left(\frac{A_{4}}{B_{4}}\right) \epsilon_{1}+\left(\frac{A_{5}}{B_{5}}\right) \epsilon_{2},
\end{aligned}
$$

where $A_{i}, B_{i}, i=1,2,3,4,5$ are numerator and denominator of the coefficients, which correspond to classical part, P-R drags $W_{1}$ and $W_{2}$ of the primaries, radiation pressure $\epsilon_{1}=1-q_{1}$ and $\epsilon_{2}=1-q_{2}$ of the primaries, respectively. On simplification, we found that

$$
\begin{aligned}
A_{1}= & -35+541 \omega_{1}^{2} \omega_{2}^{2}-644 \omega_{1}^{4} \omega_{2}^{4}, \\
A_{2}= & 26244(2262-653 b)- \\
& 27(5292162-4787719 b) \omega_{1}^{2} \omega_{2}^{2} \\
& -2(402982614-10430203 b) \omega_{1}^{4} \omega_{2}^{4} \\
& +32(12457908-1490819 b) \omega_{1}^{6} \omega_{2}^{6} \\
& +1024(67581+1634 b) \omega_{1}^{8} \omega_{2}^{8}, \\
A_{3}= & -78732(45) \\
& 27(3181248+4414649 s q r t 3 b) \omega_{1}^{2} \omega_{2}^{2} \\
& -6(72610776+10390609 \sqrt{3} b) \omega_{1}^{4} \omega_{2}^{4} \\
+ & 32(6808752-212191 \sqrt{3} b) \omega_{1}^{6} \omega_{2}^{6} \\
+ & 1024(36828+997 \sqrt{3} b) \omega_{1}^{8} \omega_{2}^{8}, \quad(66)
\end{aligned}
$$




$$
\begin{array}{rl}
A_{4}= & 8748(195 \sqrt{3}-584 b)- \\
2 & 2(465795 \sqrt{3}-1556744 b) \omega_{1}^{2} \omega_{2}^{2} \\
+ & 2(10722915 \sqrt{3}-11609036 b) \omega_{1}^{4} \omega_{2}^{4} \\
+ & 32(200970 \sqrt{3}-103079 b) \omega_{1}^{6} \omega_{2}^{6} \\
& -512(2565 \sqrt{3}-3217 b) \omega_{1}^{8} \omega_{2}^{8}, \\
A_{5}= & -8748(507 \sqrt{3}-688 b)+ \\
& 27(315819 \sqrt{3}-1533728 b) \omega_{1}^{2} \omega_{2}^{2} \\
& +2(3085838 \sqrt{3}+759212 b) \omega_{1}^{4} \omega_{2}^{4} \\
& -32(941526 \sqrt{3}-283835 b) \omega_{1}^{6} \omega_{2}^{6} \\
& -512(10251 \sqrt{3}+877 b) \omega_{1}^{8} \omega_{2}^{8}, \quad \\
B_{1}= & 8\left(1-4 \omega_{1}^{2} \omega_{2}^{2}\right)\left(4-25 \omega_{1}^{2} \omega_{2}^{2}\right), \quad \\
B_{2}= & 864 a b, \\
B_{3}= & B_{2}, \\
B_{4}= & 1152 a b, \\
B_{5}= & 576 a b, \\
a= & {\left[\omega_{1}^{2} \omega_{2}^{2}\left(1-4 \omega_{1}^{2} \omega_{2}^{2}\right)\right.} \\
& \left.\left(4-25 \omega_{1}^{2} \omega_{2}^{2}\right)\left(117+16 \omega_{1}^{2} \omega_{2}^{2}\right)\right], \quad(75) \\
b= & \sqrt{\left(27-16 \omega_{1}^{2} \omega_{2}^{2}\right)} .
\end{array}
$$

In the absence of perturbing parameters,

$$
D_{4}=\frac{-35+541 \omega_{1}^{2} \omega_{2}^{2}-644 \omega_{1}^{4} \omega_{2}^{4}}{8\left(1-4 \omega_{1}^{2} \omega_{2}^{2}\right)\left(4-25 \omega_{1}^{2} \omega_{2}^{2}\right)}
$$

which agree with the classical result (Deprit and Deprit-B 1967; Mever and Schmidt 1986; Kushvah et al 2007; Kishor and Kushvah 2017). In order to analyze the nonlinear stability of triangular equilibrium points in non-resonance case using ArnoldMoser theorem, we plot the determinant $D_{4}$ with respect to the mass parameter $\mu$ to insure the value of $D_{4}=K_{4}\left(\omega_{2}, \omega_{1}\right)$. From figures (2) 6) , it is clear that within the linear stability range $0<\mu<\mu_{c}$, there exists one value of mass parameter $\mu=\mu_{0}$, called the zero of $D_{4}$, at which $D_{4}$ vanish in each case. Thus, Arnold-Moser theorem fails, which insure that in non-resonance case, triangular equilibrium points of the problem under analysis are unstable in nonlinear sense within the linear stability range $0<\mu<\mu_{c}$. To see the effect of perturbing parameters, we have computed values of the zero $\left(\mu_{0}\right)$ of $D_{4}$ and critical mass ratio $\left(\mu_{c}\right)$ at different values of perturbing parameters $W_{1}, W_{2}, \epsilon_{1}, \epsilon_{2}$ and results are placed in Table-2 From Table-2, it is noticed that on increase in the values of $W_{1}, W_{1}, \epsilon_{1}$, value of critical mass $\mu_{c}$ increases but the value of $\mu_{0}$ is nonzero in each case. On the other hand, on increase in the value of $\epsilon_{2}, \mu_{c}$ decreases with nonzero $\mu_{0}$. Thus, from the figures (2 6) as well as from the Table-2, it is clear that radiation pressure and P-R drag of both the primaries affect the linear stability range of the problem significantly. The nonzero value of the zero $\left(\mu_{0}\right)$ of the determinant $D_{4}$ in the ArnoldMoser theorem under non-resonance case, insure the instability of triangular equilibrium points, within the range of stability $0<\mu<\mu_{c}$.

\section{Conclusions}

We have considered the photogravitational restricted three body problem in the presence of radiation pressure force and P-R drag of both the massive bodies, which are radiating in nature. Analysis of nonlinear stability of the triangular equilibrium points is performed in nonresonance case using Arnold-Moser theorem under the influence of four perturbing parameters in the form of P-R drags $W_{1}, W_{2}$ and mass reduction factors $q_{1}, q_{2}$, of both the primaries. First, we have normalized the Hamiltonian of the problem up to order four using Lie transform method and then Birkhoff normal form of the Hamiltonian constructed, which is necessary to apply the ArnoldMosor theorem in non-resonance case. The deter$\left.\frac{\text { artholom }}{\text { munam }}\right)_{4}$ of the Arnold-Moser theorem is computed analytically under the consideration of only linear order terms of perturbing parameters, which agree with that of Deprit and Deprit-Bartholome (1967); Mever and Schmidt (1986); Kushvah et al (2007); Kishor and Kushvah (2017) in the absence of perturbing parameters. To apply the ArnoldMoser theorem in non-resonance case, we have plotted the determinant $D_{4}$ with respect to the mass parameter $\mu$ within the stability range $0<$ $\mu<\mu_{c}$. It is observed that in presence as well as in absence of perturbing parameters, there exist a 


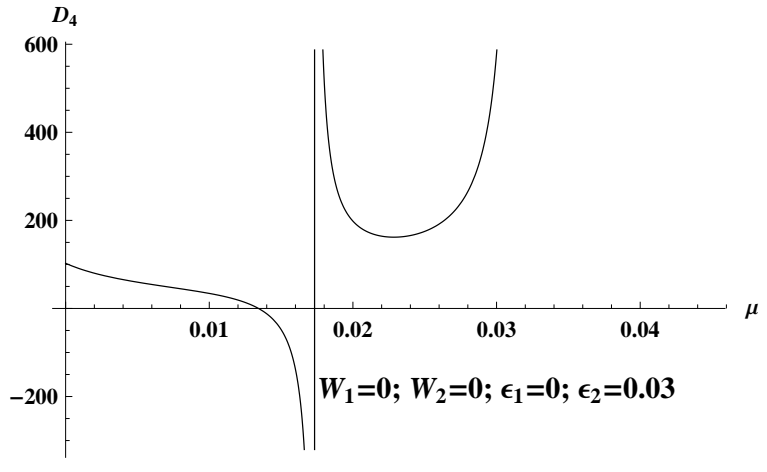

Fig. 6. - Zero $\left(\mu_{0}\right)$ of the determinant $D_{4}$ within the stability range $0<\mu<\mu_{c}$ at $\epsilon_{2}=1-q_{2}=$ $0.03, W_{1}=W_{2}=\epsilon_{1}=0$ (only in presence of radiation pressure of second primary).

Table 2: Zero $\left(\mu_{0}\right)$ of $D_{4}$ and critical mass ratio $\mu_{c}$ at different values of perturbing parameters.

\begin{tabular}{|rrrrrr|}
\hline$W_{1}$ & $W_{1}$ & $\epsilon_{1}$ & $\epsilon_{2}$ & $\mu_{0}$ & $\mu_{c}$ \\
\hline 0.000 & 0.0000 & 0.000 & 0.00 & 0.010950 & 0.0385209 \\
& & & & & \\
0.005 & 0.0000 & 0.000 & 0.00 & 0.000305 & 0.0393447 \\
0.010 & 0.0000 & 0.000 & 0.00 & 0.000876 & 0.0401684 \\
0.015 & 0.0000 & 0.000 & 0.00 & 0.005844 & 0.0409922 \\
0.020 & 0.0000 & 0.000 & 0.00 & 0.010970 & 0.0418159 \\
0.025 & 0.0000 & 0.000 & 0.00 & 0.016230 & 0.0426397 \\
& & & & & \\
0.000 & 0.0040 & 0.000 & 0.00 & 0.001122 & 0.0388504 \\
0.000 & 0.0045 & 0.000 & 0.00 & 0.000440 & 0.0388916 \\
0.000 & 0.0050 & 0.000 & 0.00 & 0.000529 & 0.0389328 \\
0.000 & 0.0055 & 0.000 & 0.00 & 0.000625 & 0.0389740 \\
0.000 & 0.0060 & 0.000 & 0.00 & 0.000727 & 0.0390152 \\
& & & & & \\
0.000 & 0.0000 & 0.001 & 0.00 & 0.014260 & 0.0385387 \\
0.000 & 0.0000 & 0.002 & 0.00 & 0.015750 & 0.0385566 \\
0.000 & 0.0000 & 0.003 & 0.00 & 0.016580 & 0.0385744 \\
0.000 & 0.0000 & 0.004 & 0.00 & 0.017320 & 0.0385922 \\
0.000 & 0.0000 & 0.005 & 0.00 & 0.017860 & 0.0386101 \\
& & & & & \\
0.000 & 0.0000 & 0.000 & 0.01 & 0.018040 & 0.0381642 \\
0.000 & 0.0000 & 0.000 & 0.02 & 0.015740 & 0.0378075 \\
0.000 & 0.0000 & 0.000 & 0.03 & 0.013380 & 0.0374508 \\
0.000 & 0.0000 & 0.000 & 0.04 & 0.011130 & 0.0370941 \\
0.000 & 0.0000 & 0.000 & 0.05 & 0.008876 & 0.0367374 \\
& & & & & \\
0.015 & 0.0050 & 0.003 & 0.031 & 0.02483 & 0.0438750 \\
\hline
\end{tabular}

nonzero value of $\mu=\mu_{0}$ at which $D_{4}$ vanish (figures (2-60), which insure that triangular equilibrium points are unstable in nonlinear sense. The effect of perturbing parameters are also analyzed and it is found that on increasing the values of $W_{1}, W_{1}, \epsilon_{1}$, critical mass ratio $\mu_{c}$ increases, with the existence of nonzero $\mu_{0}$ in each case, whereas on increasing the value of $\epsilon_{2}, \mu_{c}$ decreases with the existence of nonzero $\mu_{0}$ (Figure (11) and Table21). A similar trend is also seen in case of $\mu_{c 1}$ and $\mu_{c 2}$ (Table-1). Thus, we conclude that due to radiation pressure and P-R drag of both the primaries, the linear stability range of the problem get changed, significantly. Also, due to existence of nonzero value of the zero $\left(\mu_{0}\right)$ of the determinant $D_{4}$ in the Arnold-Moser theorem under non-resonance case, within the range of stability $0<\mu<\mu_{c}$, triangular equilibrium points are unstable in nonlinear sense. Present analysis is limited up to first order terms of the perturbing parameter, which may be extended to higher order inclusion of the terms. The results obtained can help to analyze the more generalized problem under the influence of other perturbations such as albedo, solar wind drag, Stokes drag etc.

We all are thankful to the Inter-University Center for Astronomy and Astrophysics (IUCAA), Pune for providing references through its library and computation facility in addition to local hospitality. First author is also thankful to UGC, New Delhi for providing financial support through UGC Start-up Research Grant No.-F.30$356 / 2017$ (BSR).

\section{REFERENCES}

Alvarez-Ramírez M, Skea JEF, Stuchi TJ (2015) Nonlinear stability analysis in a equilateral restricted four-body problem. Ap\&SS358:3, DOI 10.1007/s10509-015-2333-4

Birkhoff GD (1927) Dynamical System. Amer. Math. Soc. Colloq. Publ., New York

Celletti A (2010) Stability and chaos in celestial mechanics. Springer Science \& Business Media

Chernikov YA (1970) The Photogravitational Restricted Three-Body Problem. Soviet Ast.14:176 
Coppola VT, Rand RH (1988) Computer algebra, Lie Transforms and the nonlinear stability of $\mathrm{L}_{4}$. Celestial Mechanics 45:103-104, DOI 10.1007/BF01228988

Coppola VT, Rand RH (1989a) Computer Algebra Implementation of Lie Transforms for Hamiltonian Systems: Application to the Nonlinear Stability ofL4. Zeitschrift Angewandte Mathematik und Mechanik 69:275-284, DOI 10.1002/zamm.19890690903

Coppola VT, Rand RH (1989b) Computer Algebra, Lie Transforms and the Nonlinear Stability of $\mathrm{L}_{4}$. Celestial Mechanics 45:103-103

Deprit A (1969) Cannanical transformations depending on a parameter. Celestial mech 1:1-31

Deprit A, Deprit-Bartholome A (1967) Stability of the triangular Lagrangian points. AJ72:173173, DOI 10.1086/110213

Gómez G, Jorba A, Masdemont A, Simó C (1998) Study of the transfer between halo orbits. Acta Astronautica 43:493-520, DOI 10.1016/ S0094-5765(98)00177-5

Goździewski K (1998) Nonlinear Stability of the Lagrangian Libration Points in the Chermnykh Problem. Celestial Mechanics and Dynamical Astronomy 70:41-58, DOI 10.1023/A: 1008250207046

Idrisi MJ, Ullah MS (2018) Non-collinear libration points in er3bp with albedo effect and oblateness. Journal of Astrophysics and Astronomy 39(28):1

Ishwar B (1997) Non-Linear Stability in the Generalized Restricted Three-Body Problem. Celestial Mechanics and Dynamical Astronomy 65:253-289

Ishwar B, Sharma JP (2012) Non-linear stability in photogravitational non-planar restricted three body problem with oblate smaller primary. Ap\&SS337:563-571, DOI 10.1007/ s10509-011-0868-6, 1109.4206

Jorba A (1999) A methodology for the numerical computation of normal forms, centre manifolds and first integrals of hamiltonian systems. Experimental Mathematics 8(2):155-195
Jorba À, Masdemont J (1999) Dynamics in the center manifold of the collinear points of the restricted three body problem. Physica D Nonlinear Phenomena 132:189-213, DOI 10.1016/ S0167-2789(99)00042-1

Jorba À, Villanueva J (1998) Numerical computation of normal forms around some periodic orbits of the restricted three-body problem. Physica D Nonlinear Phenomena 114:197-229, DOI 10.1016/S0167-2789(97)00194-2

Kishor R, Kushvah BS (2013) Linear stability and resonances in the generalized photogravitational Chermnykh-like problem with a disc. MNRAS436:1741-1749, DOI 10.1093/mnras/ stt1692

Kishor R, Kushvah BS (2017) Normalization of Hamiltonian and nonlinear stability of the triangular equilibrium points in non-resonance case with perturbations. Ap\&SS362:156, DOI 10.1007/s10509-017-3132-x

Kushvah BS, Sharma JP, Ishwar B (2007) Nonlinear stability in the generalised photogravitational restricted three body problem with Poynting-Robertson drag. Ap\&SS312:279293, DOI 10.1007/s10509-007-9688-0, arXiv:math/0609543

Kushvah BS, Kishor R, Dolas U (2012) Existence of equilibrium points and their linear stability in the generalized photogravitational Chermnykh-like problem with powerlaw profile. Ap\&SS337:115-127, DOI 10.1007/ s10509-011-0857-9, 1107.5390

Lhotka C, Celletti A (2015) The effect of Poynting-Robertson drag on the triangular Lagrangian points. Icarus250:249-261, DOI 10. 1016/j.icarus.2014.11.039, 1412.1630

Markeev AP, Sokolskii AG (1977) On the stability of periodic motions which are close to Lagrangian solutions. Soviet Ast.21:507-512

McKenzie R, Szebehely V (1981) Non-linear stability around the triangular libration points. Celestial Mechanics 23:223-229, DOI 10.1007/ BF01230727 
Meyer KR, Schmidt DS (1986) The stability of the Lagrange triangular point and a theorem of Arnold. Journal of Differential Equations 62:222-236, DOI 10.1016/0022-0396(86) 90098-7

Meyer KR, Hall GR, Offin DC (1992) Introduction to Hamiltonian Dynamical Systems and the N-Body Problem. Springer New York, DOI 10.1007/2F978-0-387-09724-4

Mishra VK, Ishwar B (2016) Diagolization of Hamiltonian in the photogravitational restricted three body problem with P-R drag. Advances in Astrophysics 1:3

Murray CD (1994) Dynamical effects of drag in the circular restricted three-body problem. 1: Location and stability of the Lagrangian equilibrium points. Icarus112:465-484, DOI 10.1006/ icar.1994.1198

Poincaré H (1881) Mémoire sur les courbes définies par une équation différentielle, I. J Math Pures Appl 7:375-422

Poynting JH (1903) Radiation in the solar system : its effect on temperature and its pressure on small bodies. MNRAS64:A1

Ragos O, Zafiropoulos FA (1995) A numerical study of the influence of the PoyntingRobertson effect on the equilibrium points of the photogravitational restricted three-body problem. I. Coplanar case. A\&A300:568-+

Ragos O, Zagouras CG (1993) On the existence of the 'out of plane' equilibrium points in the photogravitational restricted three-body problem. Ap\&SS209:267-271, DOI 10.1007/BF00627446

Raj MXJ, Ishwar B (2017) Diagolization of Hamiltonian in the photogravitational restricted three body problem with P-R drag. International journal of Advanced Astronomy 5:2, DOI 10.14419/ijaa.v5i2.7931

Robertson HP (1937) Dynamical effects of radiation in the solar system. MNRAS97:423

Schuerman DW (1980) The restricted threebody problem including radiation pressure. ApJ238:337-342, DOI 10.1086/157989
Simó C, Gómez G, Jorba A, Masdemont J (1995) The bicircular model near the triangular libration points of the RTBP. In: Roy AE, Steves BA (eds) NATO Advanced Science Institutes (ASI) Series B, NATO Advanced Science Institutes (ASI) Series B, vol 336, pp 343-370

Singh J, Omale SO (2019) Combined effect of Stokes drag, oblateness and radiation pressure on the existence and stability of equilibrium points in the restricted four-body problem. Ap\&SS364:6, DOI 10.1007/s10509-019-3494-3

Subba Rao PV, Krishan Sharma R (1997) Effect of Oblateness on the Non-Linear Stability of in the Restricted Three-Body Problem. Celestial Mechanics and Dynamical Astronomy 65:291312

Ushiki S (1984) Normal forms for singularties of vector fields. Japan J Appl Math 1:1-37

Wolfram S (2003) The mathematica book. Wolfram Media

This 2-column preprint was prepared with the AAS LATEX macros v5.2. 